\title{
Computing the zero forcing number for generalized Petersen graphs*
}

Abstract: Let $G$ be a simple undirected graph with each vertex colored either white or black, $u$ be a black vertex of $G$, and exactly one neighbor $v$ of $u$ be white. Then change the color of $v$ to black. When this rule is applied, we say $u$ forces $v$, and write $u \rightarrow v$. A zero forcing set of a graph $G$ is a subset $Z$ of vertices such that if initially the vertices in $Z$ are colored black and remaining vertices are colored white, the entire graph $G$ may be colored black by repeatedly applying the color-change rule. The zero forcing number of $G$, denoted $Z(G)$, is the minimum size of a zero forcing set. In this paper, we investigate the zero forcing number for the generalized Petersen graphs (It is denoted by $P(n, k)$ ). We obtain upper and lower bounds for the zero forcing number for $P(n, k)$. We show that $Z(P(n, 2))=6$ for $n \geq 10, Z(P(n, 3))=8$ for $n \geq 12$ and $Z(P(2 k+1, k))=6$ for $k \geq 5$.

2010 MSC: 05C83, 05C10

Keywords: Zero forcing number, Generalized Petersen graph, Colin de Verdière parameter

\section{Introduction}

Let $G=(V, E)$ be a simple undirected graph. Each vertex is colored either white or black. In such a case we say that $G$ has a coloring and the set of all black vertices is called an initial coloring of $G$. The color-change rule is defined as follows: if $u$ is a black vertex of $G$ and exactly one neighbor $v$ of $u$ is white, then the color of $v$ changes to black.

Given a coloring of $G$, let $A$ be the set of all black vertices of $G$. The derived coloring of $A$, denoted $\operatorname{der}(A)$, is the result of applying the color-change rule until no more changes are possible. The zero forcing set for a graph $G(Z F S)$ is an initial coloring $Z$ of $G$ such that $\operatorname{der}(Z)=G$. The zero forcing number $Z(G)$ is the minimum size of all zero forcing sets of $G$. The concept of zero forcing set indicates one

* This work was supported by Mahani Mathematical Research Center, Shahid Bahonar University of Kerman, Kerman, Iran.

Saeedeh Rashidi (Corresponding Author), Nosratollah Shajareh Poursalavati, Maryam Tavakkoli; Department of Applied Mathematics, Faculty of Mathematics and Computer, Shahid Bahonar University of Kerman, Kerman, Iran(email: saeedeh.rashidi@uk.ac.ir,salavati@uk.ac.ir,mtavakkoli1362@gmail.com). 
model of propagation in general networks. It was introduced in [4]. the associated terminology has been extended in $[5,7,11,12]$. For example according to [4] if $G$ is a path, an endpoint of $G$ is the zero forcing set for $G$. If $G$ is a cycle, each set of two adjacent vertices is a zero forcing set.

A contraction of a graph $G$ is the graph obtained by identifying two adjacent vertices of $G$, and ignoring any loops or multiple edges occurred. A minor of $G$ is a graph obtained by applying a sequence of deletions of edges, deletions of isolated vertices, and contraction of edges. A graph parameter $\zeta$ is called minor monotone if for any minor $H$ of $G, \zeta(H) \leq \zeta(G)$.

Definition $1.1([9])$. The generalized Petersen graph $P(n, k)$ is defined to be the graph with the vertex set and edge set respectively as follows

$$
\begin{aligned}
& V(P(n, k))=\left\{u_{1}, \ldots, u_{n}, v_{1}, \ldots, v_{n}\right\} \\
& E(P(n, k))=\left\{u_{i} u_{i+1}, u_{i} v_{i}, v_{i} v_{i+k}: 1 \leq i \leq n\right\} .
\end{aligned}
$$

Here, the subscripts are assumed as integers modulo $n$ such that $n \geq 5$. Note that, $P(n, k) \cong P(n, n-k)$. So, we assume $n \geq 2 k+1$.

$P(n, k)$ is a 3 -regular graph with $2 n$ vertices. The generalized Petersen graph has been studied from several points of view, such as: hamiltonicity [1, 3, 15], crossing numbers [13, 14], spectrum [10] and vertex domination [9].

In Section 2, we turn to the zero forcing number of the generalized Petersen graphs. We present an upper bound for $Z(P(n, k))$. We show that $Z(P(n, 2))=6$ for $n \geq 10, Z(P(n, 3))=8$ for $n \geq 12$ and $Z(P(2 k+1, k))=6$ for $k \geq 5$.

In Section 3, we show that $K_{k,\left[\frac{n}{k}\right]}$ is a minor of $P(n, k)$ (where $[x]$ is the maximum integer not greater than $x$ ). Using this, we conclude that:

$$
\min \left\{k,\left[\frac{n}{k}\right]\right\}+1=\mu\left(K_{k,\left[\frac{n}{k}\right]}\right) \leq \mu(P(n, k)) \leq Z(P(n, k)) .
$$

The graph parameter $\mu$ is introduced by Colin de Verdiere in 1990 [6]. It is equal to the maximum nullity among all matrices satisfying several conditions. This conditions are stated in Section 3 . It is the first parameter of Colin de Verdiere type parameters. There exist a relation between this parameter and the zero forcing number that we apply it for achieving the upper bound.

There exists a comparison between the zero forcing sets and dynamic monopolies in the last section. Note that, in all figures of this paper the vertex $v_{i}\left(u_{i}\right)$ is indicated by $[i]_{v}\left([i]_{u}\right)$.

\section{Upper bounds and equalities for $Z(P(n, k))$}

In the following theorem, we obtain an upper bound for $Z(P(n, k))$, where $k \nmid n$.

Theorem 2.1. If $n=r k+s$, then $Z(P(n, k)) \leq r(s+2)$, where $1 \leq s \leq k-1, r, s \in \mathbb{N}$.

Proof. Let $A=\left\{u_{1}, u_{2}, \cdots, u_{s+2}, u_{1+k}, u_{2+k}, \cdots, u_{s+2+k}, \cdots, u_{1+(r-1) k}, u_{2+(r-1) k}, \cdots, u_{s+2+(r-1) k}\right\}$ be an initial coloring of $P(n, k)$. The following vertices change to black by the color-change rule: $\left\{v_{2}, \ldots, v_{s+1}, v_{2+k}, \ldots, v_{s+1+k}, \ldots, v_{2+(r-1) k}, \ldots, v_{s+1+(r-1) k}\right\}$. Since, two neighbors of the vertices $u_{j}, 2+i k \leq j \leq s+1+i k$ for $0 \leq i \leq r-1$ are black and the only white neighbor of them is $v_{j}$.

We also show that the vertices $\left\{v_{1}, v_{1+k}, \ldots, v_{1+(r-1) k}\right\}$ are in $\operatorname{der}(\mathrm{A})$. Note that

$$
s+1+(r-1) k=s+r k+1-k=n+1-k \equiv 1-k(\bmod (\mathrm{n})) .
$$

We have the following adjacency: 
The vertex $v_{1}$ is the only white neighbor of the black vertex $v_{s+1+(r-1) k}$ and the vertex $v_{s+1+(r-1) k}$ forces it.

The vertex $v_{k+1}$ is the only white neighbor of the black vertex $v_{1}$ and the vertex $v_{1}$ forces it.

The vertex $v_{1+(r-1) k}$ is the only white neighbor of the black vertex $v_{1+(r-2) k}$ and the vertex $v_{1+(r-2) k}$ forces it.

Also, the color of the vertices $u_{n}, u_{k}, \ldots, u_{(r-1) k}, v_{n}, v_{k}, \ldots, v_{(r-1) k}$ change to black.

The vertex $u_{n}$ is the only white neighbor of the black vertex $u_{1}$ and is forced by it.

For $i=1, \cdots, r-1$, the vertex $u_{i k}$ is the only white neighbor of the black vertex $u_{i k+1}$ and is forced by it.

The vertex $v_{n}$ is the only white neighbor of the black vertex $v_{(r-1) k+s}$ and is forced by it.

The vertex $v_{k}$ is the only white neighbor of the black vertex $v_{n}$ and is forced by it.

For $i=2, \cdots, r-1$, the vertex $v_{i k}$ is the only white neighbor of the black vertex $v_{(i-1) k}$ and is forced by it.

Now, for $i=1, \ldots, r$ :

The vertex $u_{i k-1}$ is the only white neighbor of the black vertex $u_{i k}$ and is forced by it.

Then the vertex $v_{i k-1}$ is the only white neighbor of the black vertex $u_{i k-1}$ and is forced by it.

Also for each $t<k$, it is one counter, we have:

The vertex $u_{i k-t}$ is the only white neighbor of the black vertex $u_{i k-t+1}$ and is forced by it.

the vertex $v_{i k-t}$ is the only white neighbor of the black vertex $u_{i k-t}$ and is forced by it.

This process continues until $i k-t=i(k-1)+s+3$ and $u_{i(k-1)+s+3}$ forced by $u_{i k+s+4}$. Then $v_{i(k-1)+s+2}$ is the only white neighbor of the black vertex $u_{i(k-1)+s+3}$ and is forced by it. So, all vertives of graph became black.

In the next theorem we obtain an upper bound for $Z(P(n, k))$. This bound does not depend on $n$.

Theorem 2.2. $Z(P(n, k)) \leq 2 k+2$.

Proof. Let $A=\left\{u_{1}, u_{2}, \ldots, u_{2 k+2}\right\}$ be an initial coloring of $P(n, k)$ (see Figure 1).

The vertex $v_{j}$ is the only white neighbor of the black vertex $u_{j}$ for $2 \leq j \leq 2 k+1$. It is forced by $u_{j}$. So, $v_{j} \in \operatorname{der}(\mathrm{A})$. Now the vertex $v_{1}$ is the only white neighbor of the vertex $u_{1}$ and is forced by it.

Therefore $v_{2 k+2}$ is the only white neighbor of the black vertex $v_{k+2}$. Hence, the vertices $v_{2}, v_{3}, \ldots, v_{2 k+2}$ are in $\operatorname{der}(\mathrm{A})$. We continue by induction. Let $m \geq 2 k+2$ and the color of vertices

$$
\left\{\begin{array}{l}
u_{m}, \ldots, u_{2}, u_{1} \\
v_{m}, \ldots, v_{2}
\end{array}\right.
$$

have been changed to black. It suffices to show that the color of the vertices $u_{m+1}$ and $v_{m+1}$ change to black. Note that $m \geq 2 k+2$ hence $m \geq m+1-2 k \geq 3$. Therefore, the vertex $v_{m+1}$ is the only white neighbor of the black vertex $v_{m+1-k}$ and $u_{m+1}$ is the only white neighbor of the black vertex $u_{m}$.

Corollary 2.3. If $n=r k+s$, then $Z(P(n, k)) \leq \min \{r(s+2), 2 k+2\}$, where $1 \leq s \leq k-1$.

Remark 2.4. In [2] the authors proved that $Z(P(15 r, 2))=6$ and $Z(P(24 r, 5))=12$ for all $r \geq 1$. Also they proved Theorem 2.2 another way. They obtain the upper bound $Z(P(2 k+1, k)) \leq 6$ that we conclude this upper bound from Theorem 2.1 and obtain the equality in Theorem 2.6.

Theorem 2.5. If $n \geq 10$, then $Z(P(n, 2))=6$.

Proof. By Theorem 2.2, we have $Z(P(n, 2)) \leq 6$. Hence it suffices to show that no initial coloring of the graph with five vertices can be a zero forcing set. Let $A$ be such an initial coloring. By checking all of possible cases we show that $|\operatorname{der}(\mathrm{A})| \leq 10<2 n=|P(n, 2)|$. We have illustrated all cases, unless the trivial or similar ones, in the following figures. In each figure the white vertices are the vertices that will change to black by $A$. The set $A$ can consist some vertices of type $u_{i}$ or $v_{i}$. Therefore, the following division is considered. Note that, $r$ vertices can be belong to the inner cycle of generalized peterson graph 


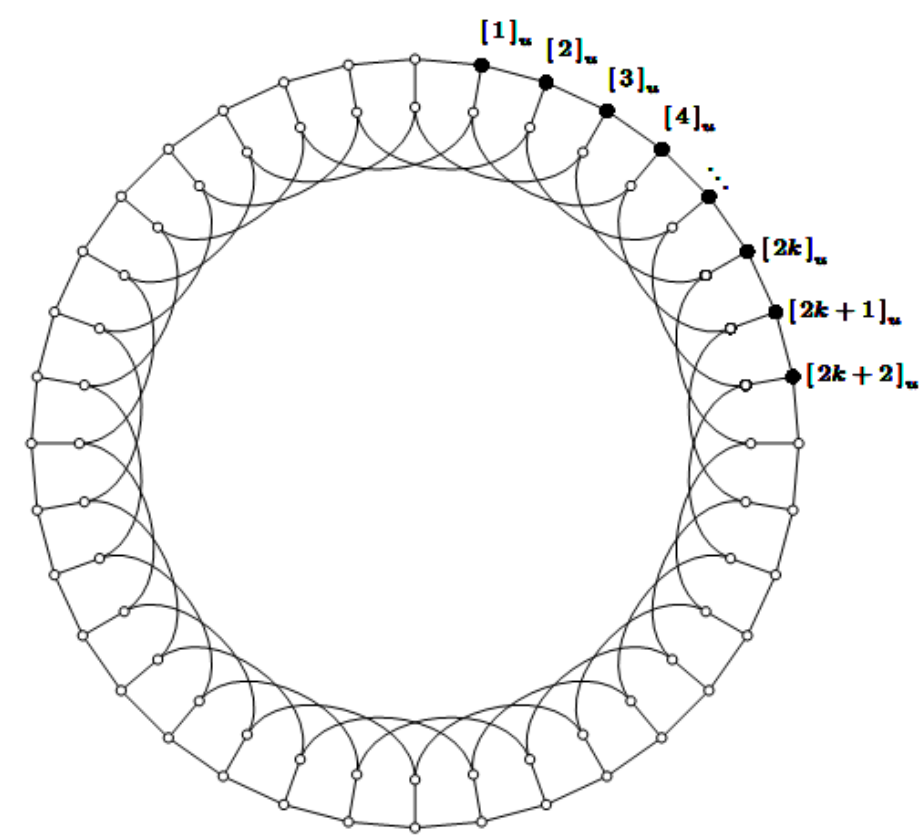

Figure 1. An initial coloring of $P(n, k)$ by $2 k+2$ vertices

and $5-r$ vertices must be belong to the outer cycle of it.

1 . The set $A$ consists of five $v$-vertices $(r=5)$ :
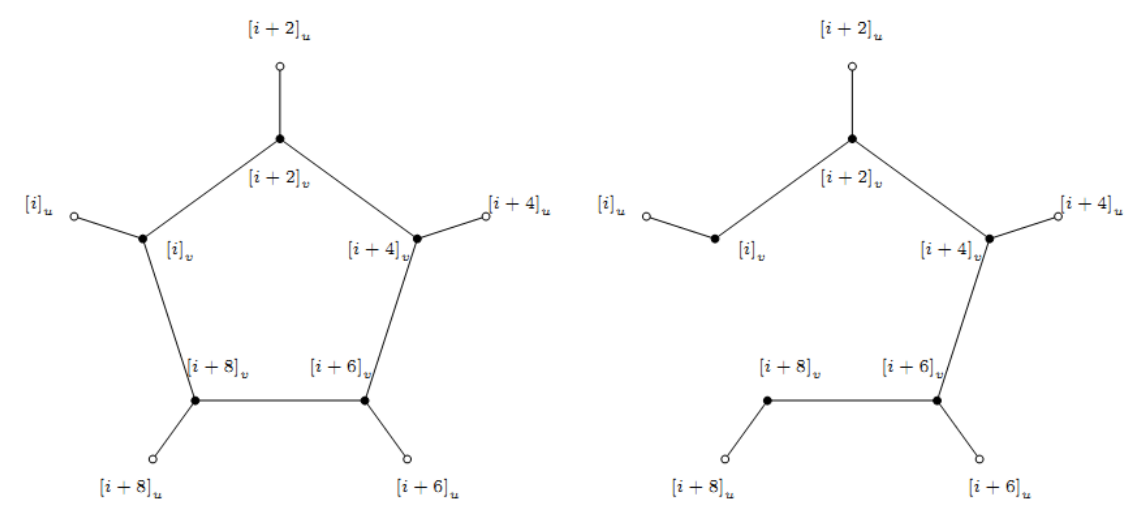

Note that, the vertex $v_{i}$ and $v_{i+8}$ are adjacent for $n=10$.

2 . The set $A$ consists of five $u$-vertices $(r=0)$ :

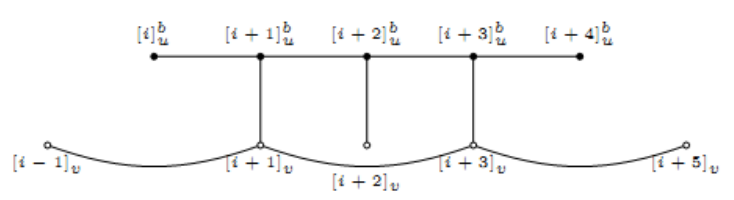


3. The set $A$ consists of four $u$-vertices and one $v$-vertex $(r=1)$ :
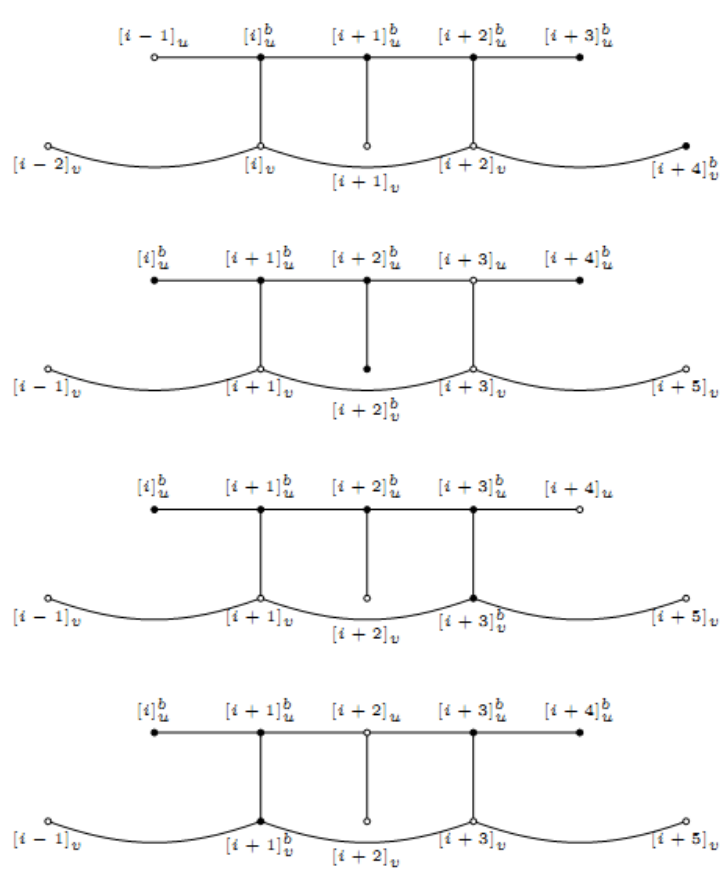

4. The set $A$ consists of four $v$-vertices and one $u$-vertex $(r=4)$ :

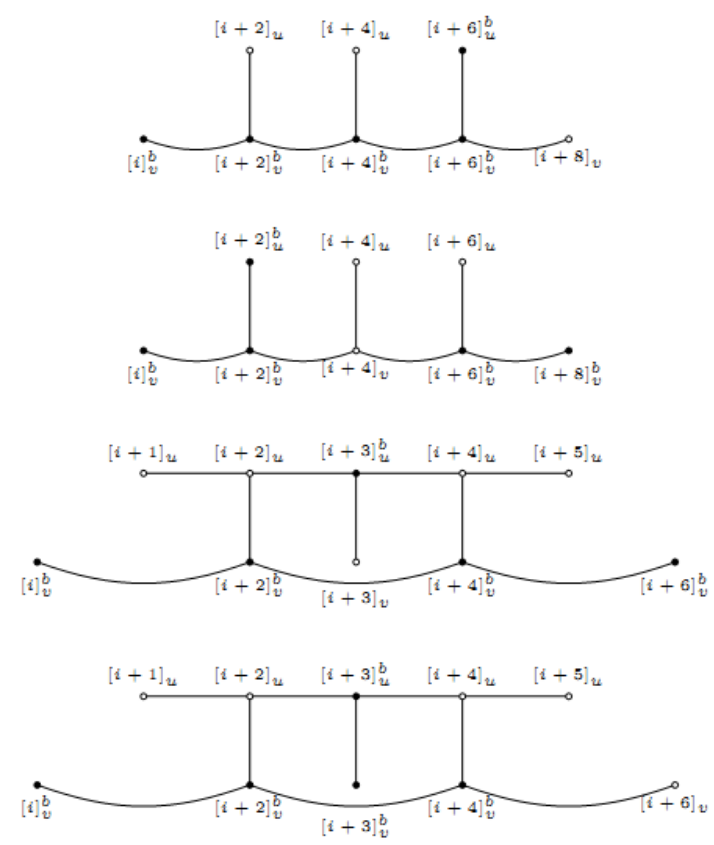

5. The set $A$ consists of three $u$-vertices and two $v$-vertices $(r=2)$ : 

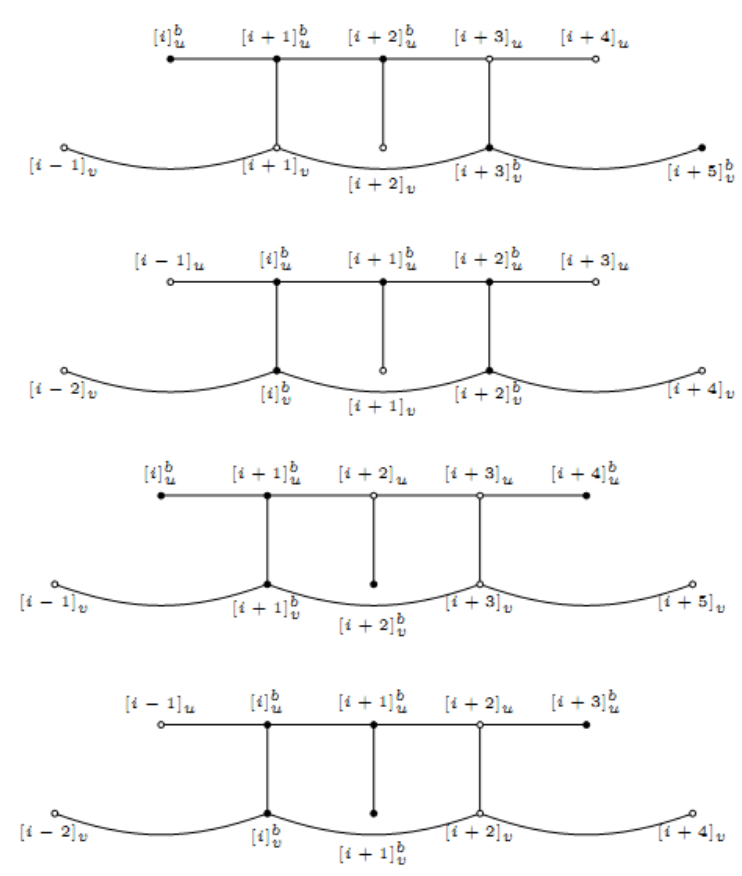

6. The set $A$ consists of two $u$-vertices and three $v$-vertices $(r=3)$ :

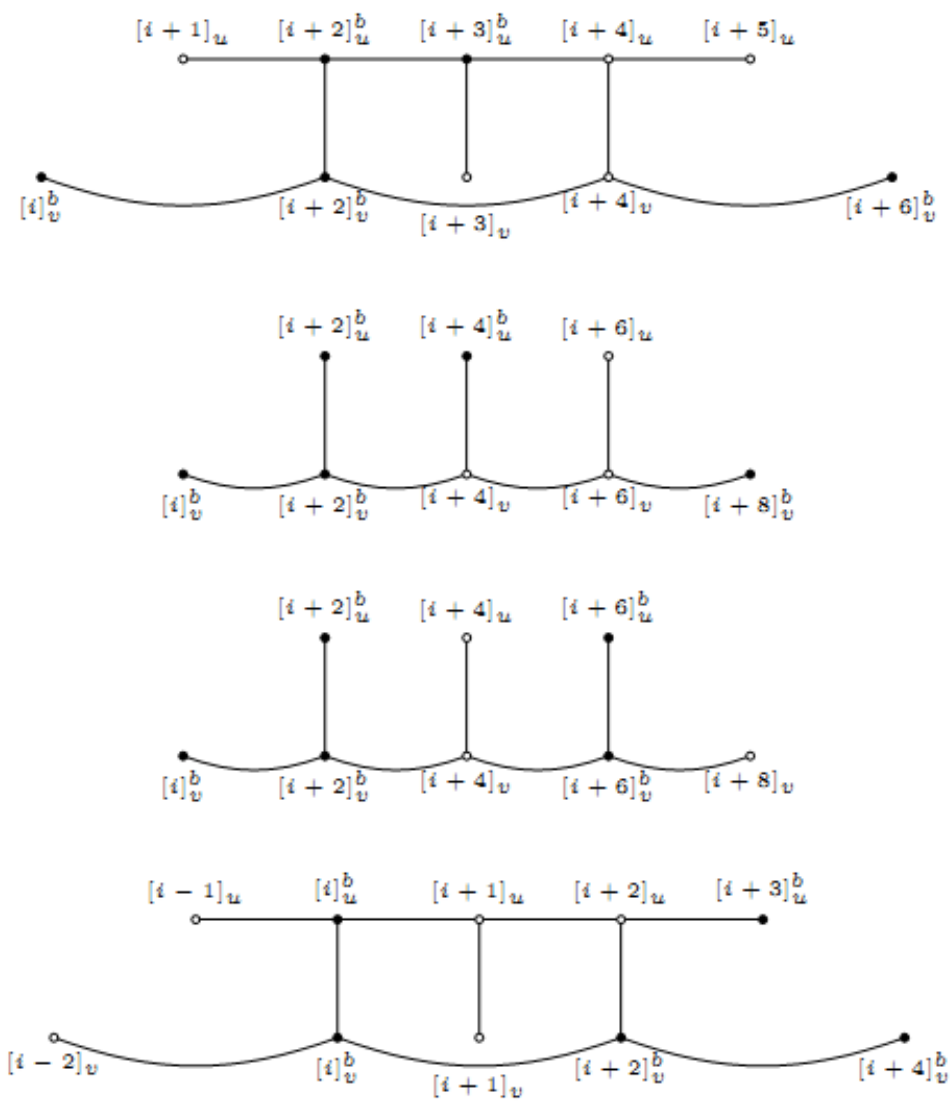


Theorem 2.6. If $k \geq 5$, then $Z(P(2 k+1, k))=6$.

Proof. By Theorem 2.1, we have $Z(P(2 k+1, k)) \leq 6$. By the same argument of Theorem 2.5, we show that, no initial coloring $A$ with 5 vertices can be a zero forcing set for $P(n, k)$. The cases are essentially same as Theorem 2.5.

1. The set $A$ consists of five $u$-vertices:

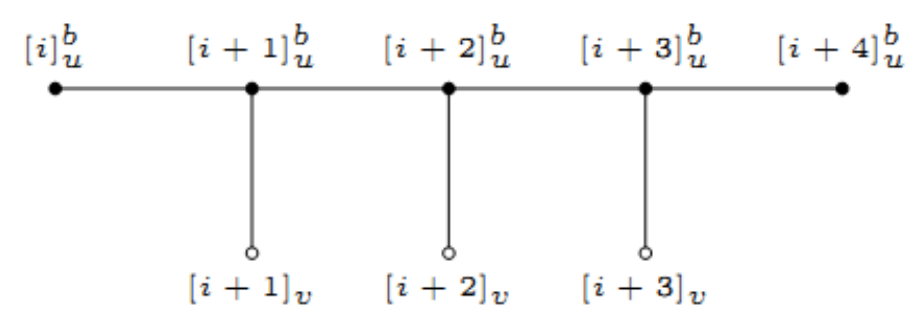

2. The set $A$ consists of five $v$-vertices:

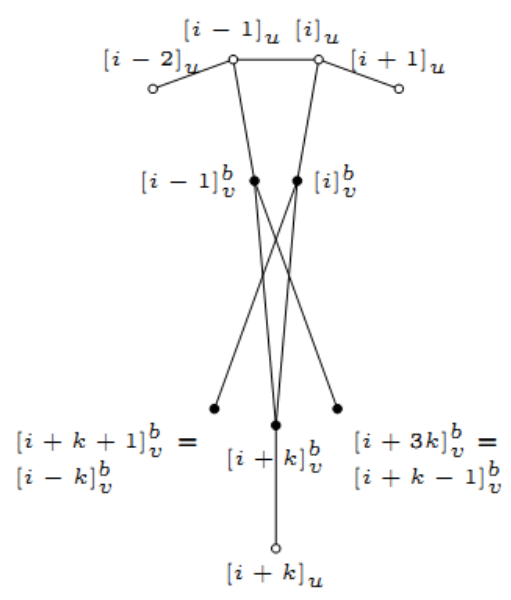

3. The set $A$ consists of four $u$-vertices and one $v$-vertex:
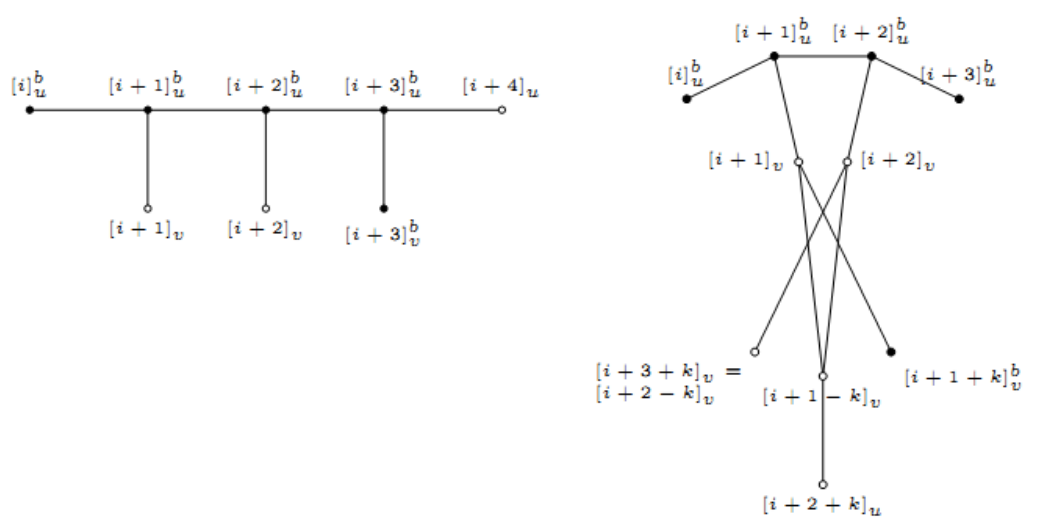
4. The set $A$ consists of four $v$-vertices and one $u$-vertex:
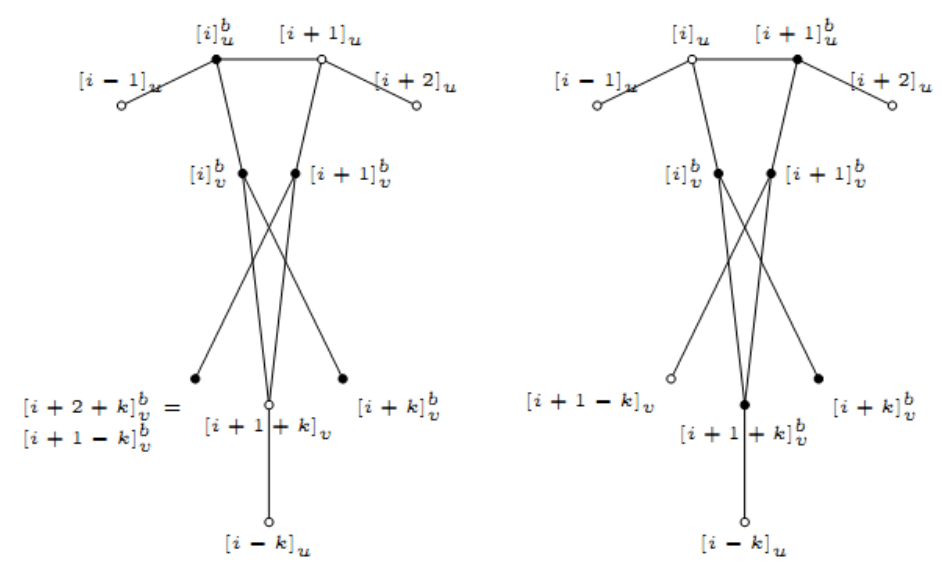

5. The set $A$ consists of three $u$-vertices and two $v$-vertices:
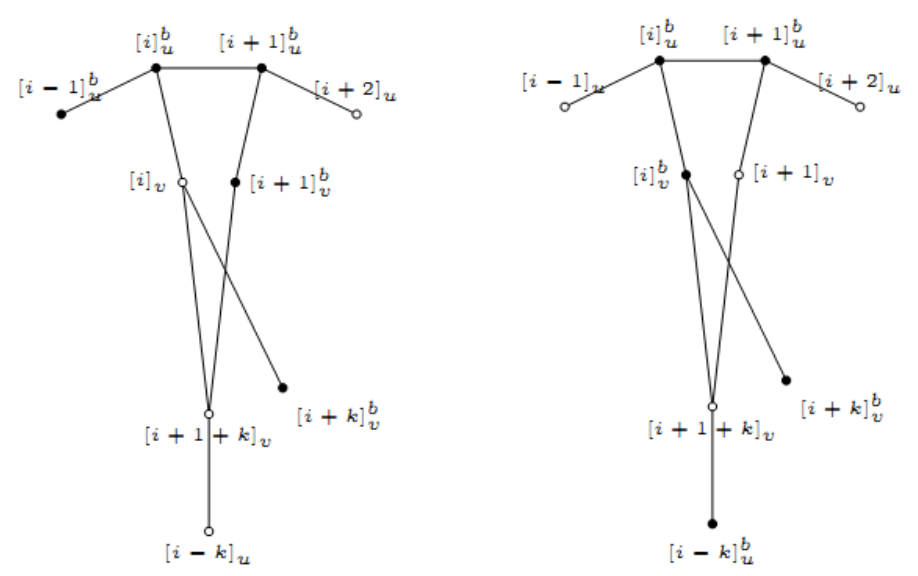

6. The set $A$ consists of two $u$-vertices and three $v$-vertices:
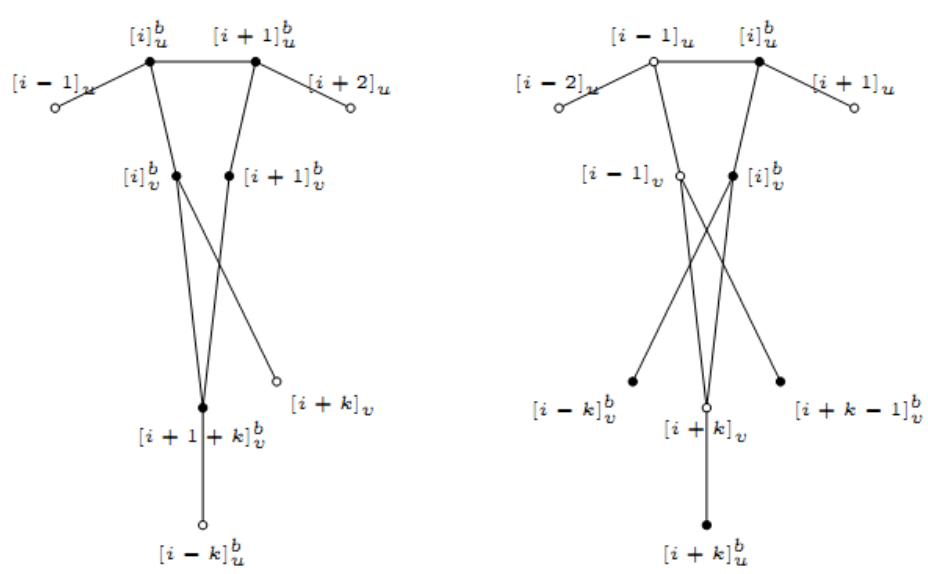


\section{Lower bound for $Z(P(n, k))$}

In this section, we obtain a lower bound for $Z(P(n, k))$. For this aim, we use the graph parameter $\mu(G)$. It has a monotonicity property, which proved first by Colin de Verdière in [8].

Definition 3.1. [16] Let $G=(V, E)$ be an undirected graph, assuming (without loss of generality) that $V=\{1, \ldots, n\}$. Then parameter $\mu(G)$ is the largest corank of any matrix $M=\left(M_{i, j}\right) \in R^{n}$ such that: (M1) for all $i, j$ with $i \neq j: M_{i, j}<0$ if $i$ and $j$ are adjacent and $M_{i, j}=0$ if $i$ and $j$ are nonadjacent;

(M2) $M$ has exactly one negative eigenvalue, of multiplicity 1 ;

(M3) there is no nonzero matrix $X=\left(X_{i, j}\right) \in R^{n}$ such that $M X=0$ and such that $X_{i, j}=0$ whenever $i=j$ or $M_{i, j} \neq 0$.

In [6] it is stated that $\mu(G) \leq Z(G)$.

Theorem 3.2. [8] If $H$ is a minor of a graph $G$, then $\mu(H) \leq \mu(G)$.

This property sometimes described as $\mu$ minor-monotone. For instance $\mu\left(K_{n}\right)=n-1$ and for $p \leq q$

$$
\mu\left(K_{p, q}\right)= \begin{cases}p & \text { if } q \leq 2 \\ p+1 & \text { if } q \geq 3\end{cases}
$$

See [16] for more details.

Definition 3.3. Let $G=(V, E)$ be a simple graph and $A, B$ be none-empty subsets of $V(G)$. We say that $A$ and $B$ are adjacent if there exist vertices $x \in A$ and $y \in B$ such that $x y \in E$. In such a case we write $A \sim B$.

Theorem 3.4. If $k \geq 3$, then the graph $K_{k,\left[\frac{n}{k}\right]}$ is a minor of $P(n, k)$.

Proof. Let $A_{i}=\left\{u_{(1+(i-1) k)}, u_{2+(i-1) k}, \ldots, u_{k+(i-1) k}\right\}$ for each $1 \leq i \leq r=\frac{n}{k}$. Put $B_{j}=$ $\left\{v_{j}, v_{j+k}, \ldots, v_{j+(r-1) k}\right\}$ for each $1 \leq j \leq k$. It is easy to see that each $A_{i}$ is adjacent to each $B_{j}$ and $B_{i}$ is not adjacent to $B_{j}$ for $i \neq j$. Now proceed as follows:

1. Delete the edges between the vertices $1+m k_{u}$ and $m k_{u}$ for each $1 \leq m \leq r$.

2. Contract all the vertices of $A_{i}$ in the vertex $u_{1+(i-1) k}$ (starting with the vertex $u_{k+(i-1) k}$ and contracting successively).

3. Contract all the vertices of $B_{j}$ in the vertex $v_{j}$.

4. Delete all the remaining vertices and their edges.

Finally, we achieve the complete bipartite graph

Now, we obtain the following lower bound.

Corollary 3.5. If $k \geq 3$, then $\min \left\{k, \frac{n}{k}\right\}+1 \leq Z(P(n, k))$.

Theorem 3.6. If $n \geq 12$, then $Z(P(n, 3))=8$.

Proof. Let $A$ be a set of initial black vertices of the graph $P(n, 3)$. By Corollary $3.5,4 \leq|A|$. Let $u_{i}$ be one white vertex on the outer cycle. The color of it can be forced by the vertex $u_{i-1}$, vertex $u_{i+1}$ or the vertex $v_{i}$. 
Assume the vertices $u_{1}, \cdots, u_{i-1} \in A$, then:

1) If the vertex $u_{i-1}$ wants to force the vertex $u_{i}$, then it is necessary that $v_{i-1} \in A$.

2) If the vertex $u_{i+1}$ wants to force the vertex $u_{i}$, then it is necessary that $v_{i+1}, u_{i+1}, u_{i+2} \in A$.

3 ) If the vertex $v_{i}$ wants to force the vertex $u_{i}$, then it is necessary that $v_{i}, v_{i-k}, v_{i+k} \in A$.

Therefor the best case for the color-change processing in the vertices of the outer cycle is that the vertex $u_{i-1}$ forces the vertex $u_{i}$. So, suppose $\left\{u_{1}, u_{2}, u_{3}, u_{4}\right\} \subseteq A$. This set can not change the color of all vertices. By a simple argument, we conclude that the set $A$ be $\left\{u_{1}, u_{2}, u_{3} \cdots, u_{8}\right\}$.

\section{A comparison between zero forcing sets and dynamic monop- olies}

In the last section, we compare the zero forcing sets with another propagation concept of graph theory. This concept is dynamic monopoly. It is studied by Zaker in [17].

Definition 4.1. [17] By a threshold assignment for the vertices of $G$ we mean any function $\tau: V(G) \rightarrow$ $N \cup\{0\}$. A subset of vertices $D$ is said to be a $\tau$-dynamic monopoly of $G$ or simply $\tau$-dynamo of $G$, if for some nonnegative integer $k$, the vertices of $G$ can be partitioned into subsets $D_{0}, D_{1}, \ldots, D_{k}$ such that $D_{0}=D$ and for any $i, 1 \leq i \leq k$, the set $D_{i}$ consists of all vertices $v$ which has at least $\tau(v)$ neighbors in $D_{0} \cup \ldots \cup D_{i-1}$. Denote the smallest size of any $\tau$-dynamo of $G$ by dyn $(G)$.

It is obvious that each $Z F S$ is a 1-dynamo. For $\tau=1$, there does not exist any resistant subgraph. So, each subgraph can be a candidate for a dynamo of graph. [17] A resistant subgraph of $G$ means each subgraph $K$ such that for each vertex $v \in K$ one has $d_{K(v)} \geq d_{G(v)} t(v)+1$, where $d_{G(v)}$ is the degree of $v$ in $G$. Zaker proved that each dynamo of graph does not contain any resistant subgraph of it [17]. So, it is satisfy for the $Z F S$.

Example 4.2. We know $Z\left(K_{n}\right)=n-1$ and $Z\left(P_{n}\right)=1$. The ZFS of complete graph $K_{n}$ and path $P_{n}$ are 1-dynamo too. For the complete graph $K_{4}$, dyn $\left(K_{4}\right) \neq Z\left(K_{4}\right)$. It is an interesting question that for what graphs there exist this equality. In this example the subsets $D_{0}$ and $D_{1}$ are $Z F S$.
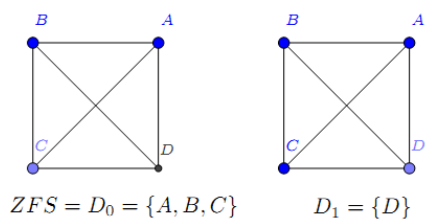

Figure 2. $\quad Z\left(K_{4}\right)=3$ and $\operatorname{dyn}\left(K_{4}\right)=1$

If we consider $D_{0}=\{A\}$ and $D_{1}=\{B, C, D\}$. Then the subset $D_{0}$ is a dynamo and it is not a $Z F S$.

There exists another question. A dynamo under what condition is a $Z F S$ ? The following lemma states this conditions.

Lemma 4.3. Consider one Dynamo as $D_{0}, D_{1}, \ldots, D_{k}$. If for each vertex $u \in D_{i+1}$ there exist a vertex $v \in D_{i}$ such that $N(v)-\{u\} \subseteq\left(D_{0} \cup D_{1} \cup \cdots \cup D_{i}\right)$, then that dynamo is a $Z F S$.

There exists one lower bound for $Z(G)$ which is obtained from the following results about dynamos.

Theorem 4.4. [17] Let $D$ be a dynamic monopoly of size $k$ in $G$. Set $H=G \backslash D$ and let $t_{\max }$ be the maximum threshold among the vertices of $H$. Then:

1) $\sum_{v \in H} t(v) \leq|E(G)|-|E(G[D])|-\delta(G)+t_{\max }$.

2) $\sum_{v \in H} t(v) \leq|E(G)|$ provided that $t(v) \leq d_{G(v)}$ for any vertex $v \in H$. 
We know that each $Z F S$ is a 1-dynamo. So, we have the following corollary.

Corollary 4.5. Let $G$ be a graph with $1 \leq \delta(G)$, then:

1) $|G|-|E(G)|+|E(G[Z F S])|+\delta-1 \leq Z(G)$.

2) $|G|-|E(G)| \leq Z(G)$

Also, Corollary 2 from [17] confirms the second inequality. This first bound is equality for some graphs. For example, let $G$ be a complete graph $K_{n}$. So $|G|-|E(G)|+|E(G[Z F S])|+\delta-1=n-$ $\frac{(n)(n-1)}{2}+\frac{(n-1)(n-2)}{2}+(n-1)-1=n-1$ and $Z\left(K_{n}\right)=n-1$. Also, it is equality for path $\left(Z\left(P_{t}\right)=1\right)$. The characterization of all graphs that satisfy this bound will be interesting.

\section{References}

[1] B. Alspach, The classification of hamiltonian of generalized Petersen graphs, J. Combin. Theory Ser. B 34(3) (1983) 293-312.

[2] J. S. Alameda, E. Curl, A. Grez, L. Hogben, O. Kingston, A. Schulte, D. Young, M. Young, Families of graphs with maximum nullity equal to zero-forcing number, Spec. Matrices 6 (2018) 56-67.

[3] K. Bannai, Hamiltonian cycles in generalized Petersen graphs, J. Combin. Theory Ser. B 24(2) (1978) $181-188$.

[4] AIM Minimum Rank - Special Graphs Work Group: F. Barioli, W. Barrett, S. Butler, S. M. Cioabă, D. Cvetković, S. M. Fallat, C. Godsil, W. Haemers, L. Hogben, R. Mikkelson, S. Narayan, O. Pryporova, I. Sciriha, W. So, D. Stevanović, H. van der Holst, K. Vander Meulen, A. Wangsness, Zero forcing sets and the minimum rank of graphs, Linear Algebra Appl. 428(7) (2008) 1628-1648.

[5] F. Barioli, W. Barrett, S. Fallat, H. T. Hall, L. Hogben, H. van der Holst, B. Shader, Zero forcing parameters and minimum rank problems, Linear Algebra Appl. 433(2) (2010) 401-411.

[6] F. Barioli, W. Barrett, S. M. Fallat, H. T. Hall, L. Hogben, B. Shader, P. van den Driessche, H. Van Der Holst, Parameters related to tree-width, zero forcing, and maximum nullity of a graph, J. Graph Theory 72(2) (2013) 146-177.

[7] F. Barioli, S. Fallat, D. Hershkowitz, H. T. Hall, L. Hogben, H. van der Holst, B. Shader, On the minimum rank of not necessarily symmetric matrices: a preliminary study, Electron. J. Linear Algebra 18 (2009) 126-145.

[8] Y. Colin de Verdière, Sur un nouvel invariant des graphs et un critère de planarité, J. Combin. Theory Ser. B 50 (1990) 11-21.

[9] J. Ebrahimi, B. N. Jahanbakhsh, E. S. Mahmoodian, Vertex domination of generalized Petersen graph, Discrete Math. 309(13) (2009) 4355-4361.

[10] R. Gera, P. Stănică, The spectrum of generalized Petersen graphs, Australian Journal of Combinatorics, 49 (2011) 39-45.

[11] L. Hogben, Minimum rank problems, Linear Algebra Appl. 432(8) (2010) 1961-1974.

[12] L. H. Huang, G. J. Chang, H. G. Yeh, On minimum rank and zero forcing sets of a graph, Linear Algebra Appl. 432(11)( (2010) 2961-2973.

[13] D. McQuillan, R. B. Richter, On the crossing numbers of certain generalized Petersen graphs, Discrete Math. 104(3) (1992) 311-320.

[14] G. Salazar, On the crossing numbers of loop networks and generalized Petersen graphs, Discrete Math. 302(1-3) (2005) 243-253.

[15] A. J. Schwenk, Enumeration of Hamiltonian cycles in certain generalized Petersen graphs, J. Combin. Theory Ser. B 47(1) (1989) 53-59.

[16] H. van der Holst, L. Lovász, A. Schrijver, The Colin de Verdière graph parameter, Graph Theory and Combinatorial Biology (Balatonlelle, 1996) volume 7 of Bolyai Soc. Math. Stud., pages 29-85. János Bolyai Math. Soc., Budapest, 1999.

[17] M. Zaker, On dynamic monopolies of graphs with general thresholds, Discrete Math. 312(6) (2012) $1136-1143$. 